\title{
Gastrointestinal manifestations in American minority population with COVID-19
}

\author{
Aleksandr Kalabin¹, Vishnu Raj Kumar Mani1,2, Sebastian Cristobal Valdivieso', \\ Brian Donaldson ${ }^{1}$ \\ ${ }^{1}$ Columbia University College of Physicians and Surgeons at Harlem Hospital, New York, NY, USA; \\ 2Duke University Medical Center, Durham, North Carolina, USA
}

Article received 3 August, 2021; accepted 5 October, 2021

\section{SUMMARY}

Background: The spectrum of gastrointestinal (GI) injuries by the SARS-CoV-2 remain largely unknown. Ethnicity data is missing or unspecified. We analyzed GI involvement in American minority patients with COVID-19 infection.

Methods: Retrospective study of hospitalized patients with confirmed COVID-19 in March-April 2020.

Results: 183 patients included: 114 (62.30\%) AfricanAmericans, 58 (31.69\%) Hispanics and 11 (6.01\%) Asians. 73 females, 110 males; mean age 64.77, mean BMI 29.03 (50.82\%); GI manifestations upon presentation: anorexia $(29.51 \%)$, diarrhea $(22.40 \%)$, nausea/ vomiting $(18.03 \%)$, abdominal pain $(9.84 \%)$. No difference observed between three ethnical groups for GI symptoms and liver function tests. $C$ Reactive Protein (CPR) $(\mathrm{P}=0.008)$, Lactate $(\mathrm{P}=0.03)$ and Prothrombin Time $(\mathrm{PT})(\mathrm{P}=0.03)$ were significantly elevated in patients without GI symptoms. No difference was observed for other laboratory tests. Patients with severe disease course/intubated had higher levels of Aspartate Transaminase (AST) (109.17 vs 53.97, $\mathrm{P}=0.018)$,
Alanine Transaminase (ALT) (79.53 vs $40.03, \mathrm{P}=0.02$ ) and total bilirubin $(0.82$ vs $0.60, \mathrm{P}=0.03)$ vs non-intubated patents as well as body temperature (101.38 vs $100.70, \mathrm{p}=0.0006), \mathrm{CRP}(24.06 \mathrm{v} 15.96, \mathrm{P}=0.019)$ and lactate $(3.28$ vs $2.13, \mathrm{P}=0.009)$. There was no correlation between severity of the disease and GI symptoms, PT, platelets and albumin. However, CRP and lactate were markedly elevated in deceased vs survived patients: (27.09 vs $16.39, \mathrm{P}=0.008)$ and ( 3.33 vs $2.10 \mathrm{P}=0.005)$ respectively.

Conclusions: 50\% of patients presented with GI symptoms and they had lower levels of inflammatory markers, better liver synthetic function, indicating less overall inflammatory response and direct viral damage. Our results suggest that SARS-CoV-2 virus targets GI tract along with the lung tissue, and the degree of hepatocyte damage correlated well with more severe disease.

Keywords: Gastrointestinal manifestations, COVID-19, American minority patients, liver injury, liver function tests.

\section{INTRODUCTION}

$\mathrm{D}$ irect viral damage of organs and systems and subsequent inflammatory cascade is considered to be a major contributor to the pathogenesis of SARS-CoV-2 infection. Lung is contemplated to be the primary organ targeted by the virus. Reportedly gastrointestinal tract and liver have also

Corresponding author

Aleksandr Kalabin

E-mail: kalabin.al@gmail.com been affected during the course of the disease. Although the data shedding light on gastrointestinal manifestations in COVID-19 patients was recently published, the spectrum of GI injury by the virus and its impact on disease course and prognosis remains largely unknown [1]. Moreover, ethnicity data on GI manifestations is missing or unspecified. On the other hand, underestimation of gastrointestinal involvement could affect diagnostic accuracy and subsequently delay treatment.

Nevertheless, research focusing on American minority population with accompanying healthcare 
disparities, differences in socioeconomic status and proved disproportionate burden of COVID-19 related outcomes may add information to the overall picture of the current pandemic and deserves additional attention [2].

Hence, the goal of our study was to investigate how COVID-19 affects gastrointestinal system and liver function in American minority patients admitted to our institution and confirmed to have SARS-CoV-2 infection.

\section{MATERIALS AND METHODS}

\section{Study design}

Ethical approval from the hospital Institutional Review Board (IRB) for Health Sciences Research was expeditiously sought and approved. This retrospective study entails the clinical and laboratory characteristics of hospitalized patients with confirmed SARS-CoV-2 infection in March - April 2020.

\section{Data collection}

Data collection was done through review of electronic medical records (EMR) for all parameters included in the study of patients confirmed with SARS-CoV-2 infection.

\section{Inclusion and exclusion criteria}

184 patients confirmed with SARS-CoV-2 infection admitted to our facility during the study period were originally included. One Caucasian patient was excluded from the final analysis.

Three main groups of patients, namely AfricanAmericans, Hispanics and Asians were initially analyzed. Patients then were classified into patients with and without gastrointestinal (GI) symptoms, and further subclassified into Nonintubated (NI-T) vs Intubated (I-T) patients and Survived (S) vs Deceased (D). Patients defined as survived were alive at the time of data accrual.

\section{Outcomes}

Primary outcome was to evaluate incidence and characteristics of GI symptoms in American minority patients with confirmed COVID-19 infection.

Secondary outcomes were to assess if GI symptoms in patients with SARS-CoV-2 infection were associated with disease severity and analyze the relationship of common biomarkers and GI manifestations.

\section{Statistical analysis}

Continuous variables were reported as mean values and standard deviation (SD), while categorical variables were expressed as count and percentage. The statistical significance of intergroup differences was compared through Analysis of Variance, Unpaired Student's t-test for continuous variables and through Pearson Chi-square $\left(\chi^{2}\right)$ test for categorical variables. $\mathrm{P}$ value $<0.05$ was considered to represent statistically significant difference. Microsoft Excel spreadsheet was used to display extrapolated data. JMP®, Version 15. SAS Institute Inc., Cary, NC, 1989-2019 was used for statistical analysis.

\section{RESULTS}

\section{Patient characteristics}

183 patients were included in our study. 114 $(62.30 \%)$ patients were African-Americans, 58 $(31.69 \%)$ were Hispanics and 11 Asians (6.01\%). Mean age of our cohort was 64.77 (SD 14.09), range 28-97. African Americans tended to be older (mean age 67.73) compared to Hispanics (mean age 61.10) and Asians (mean age 53.36) $(\mathrm{P}=0.0006)$. Gender representation was as follows: 73 females (39.89\%) and 110 males (60.11\%). Mean Body Mass index (BMI) was 29.03, range 13.38$61.68 \mathrm{~kg} / \mathrm{m}^{2}$ (SD 7.36). No significant difference was observed between ethnic groups in regards to age and BMI. Mean body temperature for the cohort was 100.89 (SD 1.68) and variance between ethnic groups was not statistically significant.

121 patients $(61.20 \%)$ reported hypertension (HTN), 82 (44.81\%) diabetes mellitus (DM), $37(20.22 \%)$ chronic kidney disease (CKD), 42 $(22.95 \%)$ coronary artery disease (CAD), 20 patients (10.93\%) bronchial asthma (BA) and 13 $(7.10 \%)$ chronic pulmonary obstructive disease (COPD). HTN in African-Americans was reported in significantly higher percentage of patients compared to Hispanics and Asians, $74.56 \%$ vs $51.72 \%$ and $54.44 \%$ respectively $(\mathrm{P}=0.009)$. Variation of other comorbidities were not statistically significant between the groups. Interestingly enough none of included in the study patients specifically reported liver of other GI problems. Mean lactate was 2.34 (SD 2.17) and mean CPR 18.45 (SD 14.16). No statistical difference for both biomarkers was observed between ethnical groups included in the study (Table 1). 
Table 1 - Baseline clinical and laboratory characteristics of ethnical groups.

\begin{tabular}{|c|c|c|c|c|c|}
\hline & $\begin{array}{c}\text { Total } \\
(n=183)\end{array}$ & $\begin{array}{c}\text { African-Americans } \\
\qquad(n=114)\end{array}$ & $\begin{array}{l}\text { Hispanics } \\
(n=58)\end{array}$ & $\begin{array}{l}\text { Asians } \\
(n=11)\end{array}$ & $P$ value \\
\hline $\begin{array}{l}\text { Age } \\
\mathrm{SD}\end{array}$ & $\begin{array}{l}64.77 \\
14.90\end{array}$ & $\begin{array}{l}67.73 \\
13.14\end{array}$ & $\begin{array}{l}61.10 \\
16.53\end{array}$ & $\begin{array}{l}53.36 \\
14.56\end{array}$ & $0.0006^{*}$ \\
\hline $\begin{array}{l}\text { Sex } \\
\text { Females } \\
\text { Males }\end{array}$ & $\begin{array}{c}73(39.89 \%) \\
110(60.11 \%)\end{array}$ & $\begin{array}{l}50(43.86 \%) \\
64(56.14 \%)\end{array}$ & $\begin{array}{l}19(43.76 \%) \\
39(67.24 \%)\end{array}$ & $\begin{array}{l}4(36.36 \%) \\
7(63.64 \%)\end{array}$ & 0.36 \\
\hline $\begin{array}{l}B M I \\
\mathrm{SD}\end{array}$ & $\begin{array}{c}29.03 \\
7.36\end{array}$ & $\begin{array}{c}29.62 \\
8.20\end{array}$ & $\begin{array}{c}28.18 \\
5.38\end{array}$ & $\begin{array}{c}27.25 \\
7.01\end{array}$ & 0.37 \\
\hline $\begin{array}{l}\text { Temperature } \\
\text { SD }\end{array}$ & $\begin{array}{c}100.89 \\
1.68\end{array}$ & $\begin{array}{c}100.80 \\
1.64\end{array}$ & $\begin{array}{c}101.08 \\
1.78\end{array}$ & $\begin{array}{c}100.82 \\
1.60\end{array}$ & 0.58 \\
\hline $\begin{array}{l}\text { Lactate } \\
\mathrm{SD}\end{array}$ & $\begin{array}{l}2.34 \\
2.17\end{array}$ & $\begin{array}{l}2.28 \\
2.28\end{array}$ & $\begin{array}{l}2.61 \\
2.08\end{array}$ & $\begin{array}{l}1.55 \\
0.49\end{array}$ & 0.39 \\
\hline $\begin{array}{l}\text { CRP } \\
\mathrm{SD}\end{array}$ & $\begin{array}{l}18.45 \\
14.16\end{array}$ & $\begin{array}{l}15.87 \\
10.44\end{array}$ & $\begin{array}{l}22.58 \\
17.28\end{array}$ & $\begin{array}{c}10.46 \\
8.49\end{array}$ & 0.053 \\
\hline $\begin{array}{l}\text { Comorbidities } \\
\text { HTN } \\
\text { DM } \\
\text { CKD } \\
\text { CAD } \\
\text { BA } \\
\text { COPD }\end{array}$ & $\begin{array}{c}121(66.12 \%) \\
82(44.81 \%) \\
37(20.22 \%) \\
42(22.95 \%) \\
20(10.93 \%) \\
13(71.04 \%)\end{array}$ & $\begin{array}{c}85(74.56 \%) \\
53(46.49 \%) \\
27(23.68 \%) \\
29(25.44 \%) \\
13(11.40 \%) \\
11(9.65 \%)\end{array}$ & $\begin{array}{c}30(51.72 \%) \\
26(44.83 \%) \\
9(15.52 \%) \\
10(17.24 \%) \\
5(8.62 \%) \\
2(3.45 \%)\end{array}$ & $\begin{array}{c}6(54.55 \%) \\
3(27.27 \%) \\
1(9.09 \%) \\
3(27.27 \%) \\
2(18.18 \%) \\
0\end{array}$ & $\begin{array}{c}0.009^{*} \\
0.47 \\
0.29 \\
0.45 \\
0.63 \\
0.21\end{array}$ \\
\hline
\end{tabular}

Notes: BMI - Body Mass Index; CRP - C Reactive Protein; HTN - Hypertension; DM - Diabetes Mellitus; CKD - Chronic kidney disease; CAD Coronary artery disease; BA - Bronchial asthma; COPD - Chronic obstructive pulmonary disease; SD - Standard Deviation;

*statistically significant value $(\mathrm{P}<0.05)$.

Table 2 - Gastrointestinal symptoms and liver function tests of ethnical groups.

\begin{tabular}{|c|c|c|c|c|c|}
\hline & Total & African-Americans & Hispanics & Asians & $P$ value \\
\hline \multicolumn{6}{|l|}{ GI symptoms } \\
\hline Anorexia & $54(29.51 \%)$ & $33(28.95 \%)$ & $17(29.31 \%)$ & $4(36.36 \%)$ & 0.88 \\
\hline Diarrhea & $41(22.40 \%)$ & $24(21.05 \%)$ & $14(24.14 \%)$ & $3(27.27 \%)$ & 0.83 \\
\hline Nausea/vomiting & $33(18.03 \%)$ & $18(15.79 \%)$ & $12(20.69 \%)$ & $3(27.27 \%)$ & 0.52 \\
\hline Abdominal pan & $18(9.84 \%)$ & $10(8.77 \%)$ & $7(12.07 \%)$ & $1(9.09 \%)$ & 0.79 \\
\hline \multicolumn{6}{|l|}{ LFTs (mean) } \\
\hline $\begin{array}{l}\text { AST } \\
\text { SD } \\
\end{array}$ & $\begin{array}{c}63.83 \\
115.87 \\
\end{array}$ & $\begin{array}{c}62.35 \\
143.72 \\
\end{array}$ & $\begin{array}{l}67.59 \\
47.70 \\
\end{array}$ & $\begin{array}{l}57.33 \\
33.15 \\
\end{array}$ & 0.95 \\
\hline $\begin{array}{l}\text { ALT } \\
\text { SD }\end{array}$ & $\begin{array}{l}47.08 \\
85.64\end{array}$ & $\begin{array}{c}43.31 \\
103.02\end{array}$ & $\begin{array}{l}53.89 \\
48.21\end{array}$ & $\begin{array}{l}47.89 \\
35.96\end{array}$ & 0.76 \\
\hline $\begin{array}{l}\text { Total bilirubin } \\
\text { SD }\end{array}$ & $\begin{array}{l}0.64 \\
0.51\end{array}$ & $\begin{array}{l}0.58 \\
0.35\end{array}$ & $\begin{array}{l}0.75 \\
0.72\end{array}$ & $\begin{array}{l}0.66 \\
0.41\end{array}$ & 0.15 \\
\hline $\begin{array}{l}\text { Albumin } \\
\text { SD }\end{array}$ & $\begin{array}{l}3.60 \\
0.52\end{array}$ & $\begin{array}{l}3.57 \\
0.51\end{array}$ & $\begin{array}{l}3.66 \\
0.50\end{array}$ & $\begin{array}{l}3.61 \\
0.74\end{array}$ & 0.55 \\
\hline $\begin{array}{l}\text { PT } \\
\text { SD }\end{array}$ & $\begin{array}{c}14.38 \\
3.46\end{array}$ & $\begin{array}{c}13.99 \\
2.55\end{array}$ & $\begin{array}{c}15.06 \\
4.64\end{array}$ & $\begin{array}{c}13.83 \\
2.05\end{array}$ & 0.46 \\
\hline $\begin{array}{l}\text { Platelets } \\
\text { SD }\end{array}$ & $\begin{array}{c}220.94 \\
83.59\end{array}$ & $\begin{array}{c}220.52 \\
83.97\end{array}$ & $\begin{array}{c}221.14 \\
88.11\end{array}$ & $\begin{array}{c}224.27 \\
56.84\end{array}$ & 0.99 \\
\hline
\end{tabular}

Notes: GI - gastrointestinal; LFT - liver function tests; AST - Aspartate aminotransferase; ALT - Alanine aminotransferase; PT - Prothrombin time; SD - Standard Deviation. 
GI symptoms

Most common GI symptom in patients with confirmed COVID-19 infection upon presentation was anorexia noted in $54(29.51 \%)$ patients. 41 patients $(22.40 \%)$ complained of diarrhea (more than 3 loose stools per day), 33 (18.03\%) had nausea or vomiting and 18 patients (9.84\%) reported abdominal pain. No statistical difference was observed between three ethnical groups in regards to presenting GI symptoms (Table 2).

\section{Liver function tests (LFTs)}

Evaluation of liver function tests on admission revealed mean aspartate transaminase (AST) of
63.83 (SD 115.87), mean alanine transaminase (ALT) 47.08 (SD 85.64). Total bilirubin for the entire cohort was 0.64 (SD 0.51), mean albumin 3.60 (SD 0.52). Analysis of prothrombin time [PT] for patients included in the study affirmed mean of 14.38 (SD 3.46). No significant difference was noted between the ethnic groups. Mean platelet count was 220.94 (83.59), with no difference between groups as well (Table 2).

\section{Primary outcomes}

Overall 93 patients $(50.82 \%)$ out of 183 [35 females $(37.63 \%)$ and $58(62.37 \%)$ males] presented with GI complaints. Those patients identified them-

Table 3 - Baseline and laboratory characteristics of patients with and without GI symptoms.

\begin{tabular}{|c|c|c|c|c|}
\hline & Total & $\begin{array}{c}\text { Patient with } \\
\text { GI symptoms }(n=93)\end{array}$ & $\begin{array}{c}\text { Patient without } \\
\text { GI symptoms }(n=90)\end{array}$ & $P$ value \\
\hline $\begin{array}{l}\text { Age } \\
\mathrm{SD}\end{array}$ & $\begin{array}{l}64.77 \\
14.90 \\
\end{array}$ & $\begin{array}{l}64.41 \\
15.21 \\
\end{array}$ & $\begin{array}{l}65.13 \\
14.65 \\
\end{array}$ & 0.74 \\
\hline $\begin{array}{l}\text { Sex } \\
\text { Females } \\
\text { Males }\end{array}$ & $\begin{array}{c}73(39.89 \%) \\
110(60.11 \%)\end{array}$ & $\begin{array}{l}35(37.63 \%) \\
58(62.37 \%) \\
\end{array}$ & $\begin{array}{l}38(42.22 \%) \\
52(57.78 \%) \\
\end{array}$ & 0.53 \\
\hline $\begin{array}{l}\text { Ethnicity } \\
\text { African Americans } \\
\text { Hispanics } \\
\text { Asians }\end{array}$ & $\begin{array}{c}114(62.30 \%) \\
58(31.69 \%) \\
11(6.01 \%)\end{array}$ & $\begin{array}{c}55(59.14 \%) \\
31(33.33 \%) \\
7(7.53 \%)\end{array}$ & $\begin{array}{c}59(65.56 \%) \\
27(30.00 \%) \\
4(4.44 \%)\end{array}$ & 0.55 \\
\hline $\begin{array}{l}B M I \\
\mathrm{SD}\end{array}$ & $\begin{array}{c}29.03 \\
7.36\end{array}$ & $\begin{array}{c}28.74 \\
7.42\end{array}$ & $\begin{array}{c}29.32 \\
7.32\end{array}$ & 0.60 \\
\hline $\begin{array}{l}\text { Temperature } \\
\text { SD }\end{array}$ & $\begin{array}{c}100.89 \\
1.68\end{array}$ & $\begin{array}{c}100.77 \\
1.65\end{array}$ & $\begin{array}{c}101.00 \\
1.72\end{array}$ & 0.35 \\
\hline $\begin{array}{l}\text { Lactate } \\
\mathrm{SD}\end{array}$ & $\begin{array}{l}2.34 \\
2.17\end{array}$ & $\begin{array}{l}1.99 \\
1.13\end{array}$ & $\begin{array}{l}2.73 \\
2.90\end{array}$ & $0.03^{*}$ \\
\hline $\begin{array}{l}\text { CRP } \\
\text { SD }\end{array}$ & $\begin{array}{l}18.45 \\
14.16\end{array}$ & $\begin{array}{l}14.23 \\
10.62\end{array}$ & $\begin{array}{l}22.67 \\
16.03\end{array}$ & $0.008^{*}$ \\
\hline $\begin{array}{l}\text { Comorbidities } \\
\text { HTN } \\
\text { DM } \\
\text { CKD } \\
\text { CAD } \\
\text { BA } \\
\text { COPD }\end{array}$ & $\begin{array}{c}121(66.12 \%) \\
82(44.81 \%) \\
37(20.22 \%) \\
42(22.95 \%) \\
20(10.93 \%) \\
13(71.04 \%)\end{array}$ & $\begin{array}{c}59(63.44 \%) \\
47(50.54 \%) \\
18(19.35 \%) \\
21(22.58 \%) \\
9(9.68 \%) \\
5(5.38 \%)\end{array}$ & $\begin{array}{c}62(68.89 \%) \\
35(38.89 \%) \\
19(21.11 \%) \\
21(23.33 \%) \\
11(12.22 \%) \\
8(8.89 \%)\end{array}$ & $\begin{array}{l}0.44 \\
0.11 \\
0.77 \\
0.90 \\
0.58 \\
0.36\end{array}$ \\
\hline $\begin{array}{l}\text { LFTs } \\
\text { AST } \\
\text { ALT } \\
\text { Total bilirubin } \\
\text { Albumin } \\
\text { PT } \\
\text { Platelets }\end{array}$ & $\begin{array}{c}63.83(115.87) \\
47.08(85.64) \\
0.64(0.51) \\
3.60(0.52) \\
14.38(3.46) \\
220.94(83.59)\end{array}$ & $\begin{array}{c}52.60(32.83) \\
39.88(36.19) \\
0.59(0.42) \\
3.59(0.54) \\
14.45(2.27) \\
228.65(80.83)\end{array}$ & $\begin{array}{c}75.32(161.17) \\
54.46(116.14) \\
0.69(0.58) \\
3.61(0.50) \\
15.21(4.11) \\
212.98(86.08)\end{array}$ & $\begin{array}{c}0.20 \\
0.27 \\
0.19 \\
0.77 \\
0.03^{*} \\
0.21\end{array}$ \\
\hline
\end{tabular}

GI - gastrointestinal; BMI - body mass index; CRP - C reactive protein; HTN - Hypertension; DM - Diabetes Mellitus; CKD - Chronic kidney disease; CAD - Coronary artery disease; BA - Bronchial asthma; COPD - Chronic obstructive pulmonary disease; LFT - liver function tests; AST - Aspartate aminotransferase; ALT - Alanine aminotransferase; PT - Prothrombin time; SD - Standard Deviation; ${ }^{*}$-statistically significant value $(\mathrm{P}<0.05)$. 
selves as African American in 55 (59.14\%) cases, as Hispanic in $31(33.33 \%)$ and 7 (7.53) patients identified themselves as Asians ( $\mathrm{P}=0.55)$.

Mean age for the groups of patients with GI manifestations was 64.41 (SD 15.21), compared to mean age of 65.13 (SD 14.65) for patients with no GI symptoms $(\mathrm{P}=0.74)$. There was no difference in BMI (28.74, SD 0.80 vs 29.32, SD 0.80; $\mathrm{P}=0.60)$ and body temperature (100.77, SD 1.65 vs 101.00 , SD 1,$72 ; \mathrm{P}=0.35$ ) of patients presented with and without GI symptoms.

No difference was observed between patients with and with no GI symptoms in regards to comorbidities.

Interestingly enough, mean $C$ reactive protein (CRP) for patients with GI symptoms was 14.23 (SD 10.62) compared to more elevated CRP in patients with no GI manifestation: 22.67 (SD 16.03), $(\mathrm{P}=0.008)$. Same was observed in regards to lac- tate with mean of 1.99 (SD 1.13) vs mean of 2.73 (SD 2.90) $(\mathrm{P}=0.03)$ for patients presented with and with no GI symptoms respectively.

Mean PT for subgroup of patients with GI symptoms was 13.45 (SD 2.27) whereas PT for subgroup of patients with no GI symptoms mean PT was 15.20 (SD 4.11), ( $\mathrm{P}=0.03)$. No difference was observed between these two subgroups in regards to AST, ALT, platelets, total bilirubin and albumin (Table 3).

\section{Secondary outcomes}

In order to assess if GI symptoms and liver function tests were correlated with disease severity we subdivided our patients in intubated versus nonintubated and survived and deceased groups.

No difference was observed between intubated versus non-intubated and between survived versus deceased patients in regards to GI symptoms.

Table 4 - Gastrointestinal symptoms and liver function tests of patients based on need for mechanical ventilation or mortality.

\begin{tabular}{|c|c|c|c|c|c|c|c|}
\hline & Total & Intubated & $\begin{array}{c}\text { Non- } \\
\text { intubated }\end{array}$ & Pvalue & Survived & Deceased & $P$ value \\
\hline \multicolumn{8}{|l|}{ GI symptoms } \\
\hline Anorexia & $54(29.51 \%)$ & $8(26.67 \%)$ & $46(30.07 \%)$ & 0.71 & $43(28.48 \%)$ & $11(34.38 \%)$ & 0.51 \\
\hline Diarrhea & $41(22.40 \%)$ & $3(10.00 \%)$ & $38(24.84 \%)$ & 0.07 & $36(23.84 \%)$ & $5(15.63 \%)$ & 0.31 \\
\hline Nausea/vomiting & $33(18.03 \%)$ & $4(13.33 \%)$ & $29(18.95 \%)$ & 0.46 & $28(18.54 \%)$ & $5(15.634 \%)$ & 0.70 \\
\hline Abdominal pain & $18(9.84 \%)$ & $2(6.67 \%)$ & $16(10.46 \%)$ & 0.52 & $15(9.93 \%)$ & $3(9.38 \%)$ & 0.92 \\
\hline \multicolumn{8}{|l|}{ LFTs (mean) } \\
\hline $\begin{array}{l}\text { AST } \\
\text { SD }\end{array}$ & $\begin{array}{c}63.83 \\
115.87\end{array}$ & $\begin{array}{l}109.17 \\
261.87\end{array}$ & $\begin{array}{l}53.97 \\
36.10\end{array}$ & $0.02^{*}$ & $\begin{array}{c}66.40 \\
126.03\end{array}$ & $\begin{array}{l}50.41 \\
21.98\end{array}$ & 0.51 \\
\hline $\begin{array}{l}\text { ALT } \\
\text { SD }\end{array}$ & $\begin{array}{l}47.08 \\
85.64\end{array}$ & $\begin{array}{c}79.53 \\
185.63\end{array}$ & $\begin{array}{l}40.03 \\
36.96\end{array}$ & $0.02^{*}$ & $\begin{array}{l}48.89 \\
92.88\end{array}$ & $\begin{array}{l}37.63 \\
23.46\end{array}$ & 0.53 \\
\hline $\begin{array}{l}\text { Total bilirubin } \\
\text { SD }\end{array}$ & $\begin{array}{l}0.64 \\
0.51\end{array}$ & $\begin{array}{l}0.82 \\
0.51\end{array}$ & $\begin{array}{l}0.60 \\
0.50 \\
\end{array}$ & $0.03^{*}$ & $\begin{array}{l}0.65 \\
0.51\end{array}$ & $\begin{array}{l}0.60 \\
0.52 \\
\end{array}$ & 0.65 \\
\hline $\begin{array}{l}\text { Albumin } \\
\text { SD }\end{array}$ & $\begin{array}{l}3.60 \\
0.52 \\
\end{array}$ & $\begin{array}{l}3.65 \\
0.61\end{array}$ & $\begin{array}{l}3.59 \\
0.50\end{array}$ & 0.58 & $\begin{array}{l}3.61 \\
0.50\end{array}$ & $\begin{array}{l}3.52 \\
0.63 \\
\end{array}$ & 0.40 \\
\hline $\begin{array}{l}\text { PT } \\
\text { SD }\end{array}$ & $\begin{array}{c}14.38 \\
3.46\end{array}$ & $\begin{array}{c}14.87 \\
2.89\end{array}$ & $\begin{array}{c}14.24 \\
3.61\end{array}$ & 0.54 & $\begin{array}{c}14.24 \\
2.82\end{array}$ & $\begin{array}{c}15.31 \\
6.51\end{array}$ & 0.39 \\
\hline $\begin{array}{l}\text { CRP } \\
\text { SD }\end{array}$ & $\begin{array}{l}18.45 \\
14.16\end{array}$ & $\begin{array}{l}24.06 \\
11.60\end{array}$ & $\begin{array}{l}15.96 \\
14.58\end{array}$ & $0.02^{*}$ & $\begin{array}{l}16.39 \\
11.07\end{array}$ & $\begin{array}{l}27.09 \\
21.49\end{array}$ & $0.008^{*}$ \\
\hline $\begin{array}{l}\text { Lactate } \\
\text { SD }\end{array}$ & $\begin{array}{l}2.34 \\
2.17\end{array}$ & $\begin{array}{l}3.28 \\
2.61\end{array}$ & $\begin{array}{l}2.13 \\
2.01\end{array}$ & $0.009^{*}$ & $\begin{array}{l}2.10 \\
1.45\end{array}$ & $\begin{array}{l}3.33 \\
3.85\end{array}$ & $0.005^{*}$ \\
\hline $\begin{array}{l}\text { Platelets } \\
\text { SD }\end{array}$ & $\begin{array}{c}220.94 \\
83.59\end{array}$ & $\begin{array}{c}223.43 \\
73.37\end{array}$ & $\begin{array}{c}220.45 \\
85.67\end{array}$ & 0.85 & $\begin{array}{c}216.69 \\
73.92\end{array}$ & $\begin{array}{c}221.84 \\
85.70\end{array}$ & 0.75 \\
\hline
\end{tabular}

Notes: GI - gastrointestinal; LFT - liver function tests; AST - Aspartate aminotransferase; ALT - Alanine aminotransferase; PT - Prothrombin time; CRP - C Reactive Protein; SD - Standard Deviation; *-statistically significant value $(\mathrm{P}<0.05)$. 
Intubated patients tended to have higher levels of AST (109.17 vs 53.97, P=0.018), ALT (79.53 vs 40.03, $\mathrm{P}=0.02)$ and total bilirubin (0.82 vs 0.60 , $\mathrm{P}=0.03$ ) compared to non-intubated patents.

These liver enzymes correlated well with body temperature that was significantly higher, i.e. 101.38 for intubated group compared to non-intubated patients $100.70(\mathrm{p}=0.0006)$, as well as CRP $(24.06 \mathrm{v}$ $15.96, \mathrm{P}=0.019)$ and lactate ( 3.28 vs $2.13, \mathrm{P}=0.009)$.

No difference between groups was observed for PT, platelets and albumin.

Comparison of GI symptoms and liver function tests for survived vs deceased groups revealed no difference.

Same trend was observed for the CRP and lactate in deceased patients. They were significantly elevated compared to the survived group: (27.09 vs $16.39, \mathrm{P}=0.008)$ and (3.33 vs $2.10 \mathrm{P}=0.005)$ respectively. All other LFT's were not statistically significant (Table 4).

\section{DISCUSSION}

It has been established that Coronavirus gains its entry into the host cells by binding to the angiotensin-converting enzyme 2 (ACE2) receptor [3]. Virulence and high rate of transmission could be explained by the high affinity of the Sars-CoV-2 virus to the receptor [4-13]. Lungs are the primary target for the virus due to the high expression of ACE2 receptors on the type II alveolar epithelial cells allowing the virus to enter the cell and to replicate. Although heterogenous molecular expression of the ACE2 receptors on lung cells in different populations was recently reported by Zhao et al., more studies are needed to validate this [5]. More importantly, ACE2 receptors are also expressed on the cells of gastrointestinal tract - differentiated enterocytes, and this represents an alternative entry site for the virus with its own implications [6, 7]. Moreover, expression on digestive tract cells could even be higher than in the lung, providing additional route for SARS-CoV-2 infection and outlining disease phenotype $[8,11$, 12]. Even though Paisiz et al. recently reported significantly increased ACE2 receptor expression with associated liver injury as a result of hepatocellular hypoxia, no studies to date have revealed if ethnic differences exist [9].

In our study more than $50 \%$ of patients with confirmed COVID-19 presented with GI symptoms most likely confirming significant ACE2 receptor expression on the cells of GI tract and enterocytes representing an additional entry site for the virus. More importantly we did not observe any statistical difference between three ethnical groups in regards to presenting GI symptoms, degree of hepatocellular injury and liver synthetic function, perhaps indicating comparable molecular expression of ACE2 receptors on the GI cells of all minority patients.

Direct correlation of conventional inflammatory markers with degree of severity in COVID-19 patients was recently reported [10]. In our study patients with more severe disease course and thus requiring mechanical ventilation were noted to have higher body temperature, CRP and lactate. Biomarkers of liver cell damage tended to be higher in intubated subgroup of patients as well, confirming association of liver damage and severe disease trajectory in patients requiring mechanical ventilation.

Interestingly enough CRP and lactate were both significantly lower in group of people presented with GI symptoms. Therefore, the interpretation of this data points towards a protective effect of GI involvement in the clinical course of COVID-19 infection, as demonstrated by decreased inflammatory markers.

Liver synthetic function was also less affected if patients who had GI manifestations on presentation, again substantiating our theory of less direct viral damage and conceivably better prognosis if both respiratory and GI systems were involved.

The consequences of GI tract involvement in patients with COVID-19 infection could have an important impact in management and serve as a potential predictor of disease severity [14, 15]. However, further research is needed to validate our conclusions and we urge health care workers to include assessment of GI symptoms in patient with COVID-19.

Our study is not without limitations. It is a retrospective cohort study conducted in a single institution, the sample size Hispanics and Asians was fairly small. We would also like to acknowledge that laboratory values were taken at a set point in time and each individual patient could have been at different stage of the disease spectrum. Other environmental factors could have influenced observed laboratory derangements and it is essential to consider this while interpreting our results. 
Due to overwhelming nature of the pandemic imaging studies of gastrointestinal tract organs were done selectively and were not included into final analysis.

\section{CONCLUSIONS}

Our study is the first to date to report GI manifestations and biochemical confirmation of liver injury in American minority population with confirmed COVID-19 infection. Patients with GI symptoms tended to have lower levels of inflammatory markers and better liver synthetic function indicating less overall inflammatory response and direct viral damage which could be potentially protective in the long term. On the other hand, our results suggest that SARS-CoV-2 virus targets GI tract and liver parenchymal cells as well as lung tissue, and the degree of liver cells damage correlated well with more severe disease course.

The consequences of GI tract involvement in patients with COVID-19 infection could have an important impact in management and serve as a potential predictor of disease severity. However, more studies are needed to extrapolate our data on other ethnical and social-economical groups and validate our conclusions.

\section{Conflict of interest}

None.

\section{Funding}

This research did not receive any specific grant from funding agencies in the public, commercial, or non-for-profit sectors.

\section{Acknowledgments}

We would like to acknowledge and thank the Infections Diseases Department and Department of Surgery at Columbia University College of Physicians and Surgeons at Harlem Hospital.

\section{REFERENCES}

[1] Lee IC, Huo TI, Huang YH. Gastrointestinal and liver manifestations in patients with COVID-19. J Chin Med Assoc. 2020: 83 (6), 521-3. doi: 10.1097/ JCMA.0000000000000319;

[2] Webb Hooper M, Nápoles AM, Pérez-Stable EJ. CO-
VID-19 and Racial/Ethnic Disparities. JAMA. 2020; 323 (24):2466-7. doi: 10.1001/jama.2020.8598;

[3] Hoffmann M, Kleine-Weber H, Schroeder S, et al. SARS-CoV-2 Cell Entry Depends on ACE2 and TMPRSS2 and Is Blocked by a Clinically Proven Protease Inhibitor. Cell. 2020;181 (2), 271-80.e8. doi: 10.1016/j. cell.2020.02.052;

[4] Zhang H, Penninger JM, Li Y, et al. Angiotensinconverting enzyme 2 (ACE2) as a SARS-CoV-2 receptor: molecular mechanisms and potential therapeutic target. Intensive Care Med. 2020; 46 (4), 586-90. doi: 10.1007/s00134-020-05985-9;

[5] Zhao Y, Zhao Z, Wang Y, et al. Single-Cell RNA Expression Profiling of ACE2, the Receptor of SARSCoV-2. Am J Respir Crit Care Med. 2020; 202 (5), 756-9. doi: 10.1164/rccm.202001-0179LE;

[6] Lamers MM, Beumer J, van der Vaart J, e al. SARSCoV-2 productively infects human gut enterocytes. Science. 2020; 369 (6499), 50-4. doi: 10.1126/science.abc1669. [7] Wong SH, Lui RN, Sung JJ. Covid-19 and the digestive system. J Gastroenterol Hepatol. 2020; 35 (5), 744-8. doi: 10.1111/jgh.15047;

[8] Xu J, Chu M, Zhong F, et al. Digestive symptoms of COVID-19 and expression of ACE2 in digestive tract organs. Cell Death Discov. 2020 Aug 11; 6, 76. doi: 10.1038/ s41420-020-00307-w;

[9] Paizis G, Tikellis C, Cooper ME et al. Chronic liver injury in rats and humans upregulates the novel enzyme angiotensin converting enzyme 2. Gut. 2005; 54 (12), 1790-6. doi: 10.1136/gut.2004.062398;

[10] Mani VR, Kalabin A, Valdivieso SC, et al. New York Inner City Hospital COVID-19 Experience and Current Data: Retrospective Analysis at the Epicenter of the American Coronavirus Outbreak. J Med Internet Res. 2020; 22 (9), e20548. Published 2020 Sep 18. doi: 10.2196/20548;

[11] Galanopoulos M, Gkeros F, Doukatas A, et al. COVID-19 pandemic: Pathophysiology and manifestations from the gastrointestinal tract. World J Gastroenterol. 2020; 26 (31), 4579-88. doi: 10.3748/wjg.v26.i31.4579; [12] Xiao F, Tang M, Zheng X, Liu Y, Li X, Shan H. Evidence for Gastrointestinal Infection of SARSCoV-2. Gastroenterology. 2020; 158, 1831-3.e3;

[13] Patrì A, Pinchera B, Spirito L, et al. Gastrointestinal tract diseases as a risk factor for SARS-CoV-2 rectal shedding? An Italian report on 10 COVID-19 patients. Intest Res. 2021; 19 (3), 354-6.

[14] Velev V, Popov M, Velikov P, et al. COVID-19 and gastrointestinal injury: a brief systematic review and data from Bulgaria. Infez Med. 2020; (Suppl. 1), 37-41.

[15] Samidoust P, Samidoust A, Samadani AA, Khoshdoz S. Risk of hepatic failure in COVID-19 patients: A systematic review and meta-analysis. Infez Med. 2020; (Suppl. 1), 96-103. 